\title{
Uncovering Chinese Stereotypes and Their Influence on Adolescent Male Makeup Purchasing Behavior
}

\author{
Kelly Lau \\ Faculty of Public Relations and Advertising, Dept. of Humanities \& Social Science, \\ Beijing Normal University - Hong Kong Baptist University United International College \\ 2000 Jintong Road, Tangjiawan, Zhuhai, Guangdong Province, China \\ Tel: 86-756-362-0813Ｅ-mail: kellylau@uic.edu.hk
}

Received: October 22, 2017 Accepted: December 17, 2017 Published: December 19, 2017

doi: 10.5296/jsss.v5i1.12329ＵRL: http://doi.org/10.5296/jsss.v5i1.12329

\begin{abstract}
The consumption of makeup amongst males has not been fully explored by academics nor catered by the marketplace. Therefore, the desires of users are unsatisfied and as their existence are virtually unrecognized. Purchasing is the result of interactions between consumers and their respective societies. This qualitative study aims to reveal male Chinese adolescent makeup purchase behavior by exploring the impact of stereotyping on this emerging segment. Stereotyping contributes to the development of social categorization and can also be an external factor which influence purchasing decisions. The occurrence of makeup being stereotyped as being feminine is a key variable of this study. Findings pinpointed China as being a conservative market with strong traditional values. Not only was China an unfavorable environment in which to develop male makeup as a new life style, but stereotyping was also a strong influencer of social categorization. Even though stereotyping heavily influenced male Chinese adolescent purchasing behavior, it did so in a positive way, guiding adolescents to acknowledge their purchase desires and to explore new values in their conservative society. The lack of research into the male purchasing process was a key inspiration of this study. This study identified possible further research areas for academics and marketeers, especially in terms of male consumer behavior development.
\end{abstract}

Keywords: Chinese adolescents, Consumer decision making process, Male makeup purchase, Stereotype

\section{Introduction}

Makeup for men is a rising segment in the cosmetic industry. Not only does makeup product 
enhance and highlight the best skin condition, but it also strengthens confidence (Rieder, 2015; Ricciardelli, 2009). The trend for wearing makeup amongst male Chinese adolescent is heavily influenced by social media, where the objective of social media is to facilitate interaction in between users and speed up market growth (Zhou, 2014; de Vries, 2014). However, (Luo, 2009) concluded that Chinese culture is an obstacle to this trend. The stereotype of makeup being a feminine product was heavily rooted in Chinese culture. In addition, male make-up in Chinese society would be labelled as homosexual and offensive (Poulin-Dubois, 2002). Such contradictions have confused the male Chinese adolescent in terms of how they should interact with this changing trend within their society. It is therefore viable to investigate this subject matter further to enable a deeper understanding of male consumer behavior when purchasing makeup.

Previous research has pinpointed cosmetic product trends from psychological and socio-cultural perspectives (Hall, 2013; Poulin-Dubois, 2002; Souiden, 2009). Such perspectives have been proven to have a considerable amount of impact on consumer behavior in cosmetics. Furthermore, in comparison to different countries of different background, previous research concluded that psychological and socio-cultural factors were the dominant reasons for a change in consumer behavior in the cosmetics industry in recent decades (Weber, 2002; Kumar, 2014; Morphitou, 2011). Not only did they focus on makeup products but also the remaining products in the cosmetics industry. Previous research has further extended the study to include male cosmetic consumption patterns in conservative societies and implications for online promotional strategies. They highlighted male consumer attitudes and motivations towards cosmetics products from a psychological and socio-cultural perspective (Souiden, 2009; Hall, 2013). Furthermore, investigation of the information collection stage prior to the purchase of male cosmetic products pinpointed the need for further study (Khan, 2017). Khan has proven that the Chinese and Pakistani markets are underdeveloped in terms of the consumption of male cosmetic products, the major obstacle being males collecting and sharing product information prior to purchase. With this in mind, the male consumption of makeup products in conservative societies will be the focus of this study. Furthermore, the challenges the male Chinese adolescent faces and subsequent consumer behavior will be investigated.

The psychological and socio-cultural factors influencing the cosmetics industry have been critically investigated by a multitude of academic disciplines. However, the scope of investigation has neither focused on male makeup products nor the male Chinese adolescents market. Even though psychological and socio-cultural factors have proved significant in explaining consumer motivation, a greater understanding of the negative factors is required (Dahl, 2014). Negative factors are just as likely to be the result of psychological and socio-cultural influences on consumer behavior. Stereotyping is also an indicator and result of socio-cultural influences (Roest, 2009; Cuddy, 2015). Generally it is a form of label which indicates conformity to certain behavior in society; it could, however, encourage as well as discourage purchase decisions. An investigation of stereotyping is critical to understanding male cosmetic consumer behavior in the conservative Chinese market.

According to previous publications, the purchase of male cosmetic products was not the norm 
in conservative societies including China (Rieder, 2015; Khan, 2017). They pinpointed the difficulties experienced during product enquiry and its consequences for market development. Due to the sensitive nature of this subject, social media is unable to facilitate information exchange. Thus, the desire for makeup amongst male consumers was hard to stimulate in conservative markets. In this study, it is crucial to identify the role stereotyping played in the male cosmetic market from the outset. This is so as to uncover influences from both psychological and socio-cultural perspectives. This study moves on to evaluate the relationship between stereotyping and the desire for male cosmetic products. Finally, the study investigates how stereotyping impacts the information search and share stage when purchasing male cosmetic products in the Chinese adolescent market.

A trend for male makeup has developed in a variety of western countries; it is seen as an art form, a civil presentation as well as an enhancement of self-confidence. The previous study revealed psychological and socio-cultural factors influencing growth of male cosmetic consumption (Banse, 2010; Cuddy, 2015). The conflict between the traditional view of a man and male makeup as a new trend cannot be ignored. As culture is continuously changing in every society, the acceptance of new norms is difficult to measure and quantify (Leung, 2015; $\mathrm{Li}, 2009)$. Stereotyping however is a socio-cultural factor and a well-established academic concept. It is beneficial to evaluate this conflict with stereotyping as the focus point. Therefore, with the reference from western countries and previous research, this paper will set stereotyping as a major area in exploring the male makeup trend in the Chinese adolescent market. The aim of this study is to deepen the understanding of the behavior of adolescent males in terms of how they purchase makeup. In addition, this studies aims to explore the challenges consumers face in the conservative Chinese market.

\section{Literature Review}

The male makeup segment of the Chinese adolescent market is a rising segment within the cosmetics industry; research in this area has been minimal to date. Previous research on male appearance has not focused on consumer behavior relating to male makeup; they only examined other male cosmetics products. They concluded that social norms confused the purchase of male related products, which had a psychological impact on consumer behavior (Khan, 2017; Poulin-Dubois, 2002; Rieder, 2015). Previous research provided the bedrock of this study, with psychological factors being explored so as to deepen the understanding of how consumers behave in the male adolescent makeup market. Stereotyping is a well-known academic tool used to evaluate social psychology. Not only is it a socio-cultural concept, but it is also one of the external influences of the consumer decision making process model. The consumer decision making process, a core theory in examining consumer behavior, charts the consumer psychological changes of consumers during prepurchase, purchase and post purchase stages of the buying process. Having stereotyping as a focus of this study, enables a deeper understanding of the consumer decision making process when buying male makeup products. This study aims to stress the importance of stereotyping in evaluating the behavior of male Chinese adolescents within the developing cosmetics market. 


\subsection{Stereotyping}

A stereotype is an intangible term used to refer to what is considered as acceptable behavior in a society. It stresses the sense of belongings amongst groups of like-minded people. Conforming members of different stereotypes desire to pass on their values from generation to generation (Fiske, 1998; Oakes, 1994). Therefore, they exhibit consistent values with the aim of maintaining the values which they believe in. Conversely, loose values can threaten the spirit and the existence of that stereotype (Spencer, 2016). Non-conforming group members often feel anxious when interacting with conforming group members, as their behavior does not conform to the group. When male Chinese adolescents get involved in discussing and purchasing makeup products, in a feminine stereotyped environment, they can become confused regarding which group they fall into. Previous research pinpointed the need for stereotyping to evolve to reduce anxiety levels amongst non-conforming group members (Cohen, 2005; Lee, 2011).

China is a collective and conservative society with deep rooted values, that facilitates the maintenance of stereotypes (Carter, 2006). However, with the emergence of the male makeup trend in Mainland China, the stereotype of makeup being a feminine product has been shaken (Hopkins, 2007; Rocha, 2005). The role and impact of stereotyping when male makeup is purchased will be explored. Previous research highlighted that the use of attractive models in the high quality clothing market was discriminative and overgeneralized (Luoh, 2009). Which the stereotypical attractive model being a significant indicator of product quality as a result of the need maintain conforming group members. This increased anxiety levels amongst non-conforming group members as well as leading consumers to seek out desire alternatively (Parekh, 1994). Parekh pinpointed that the physical attractiveness stereotype could be mislabeled and could have a considerable impact on the desire to purchase beauty products. Previous research has identified that there is the perception that attractive physical appearance is closely associated with high job performance. (Luoh, 2009). Luoh stated that such stereotyping had a negative impact on service expectations of consumers, confusing purchasing decisions. Therefore, stereotyping should not be ignored when trying to understand the behavior of male Chinese adolescents when buying makeup. Further evaluation concentrates on whether stereotypes confuse male Chinese adolescents when purchasing makeup or whether the traditional Chinese stereotype of makeup being feminine is changing. Not only does stereotyping influence the purchasing process, more importantly, it reveals consumer behavior resulting from social-cultural environment as changing.

\subsection{Consumer Decision Making Process}

The consumer decision making process (CDMP) model has a long history in the study of consumer behavior; it is used as a central method for evaluating how purchase decisions are made. The CDMP has five structured stages to help explain how consumers behave prior to, during and after purchase (Pellémans, 1971; Clow, 2005). Only two out of five stages will be applied to this study: need recognition and information search. Previous research has pinpointed the CDMP model as enabling a clear way to analyze consumer behavior and facilitate effective communication between marketeers and consumers (Jeddi, 2013). Male 
consumer behavior has given rise to its study (Bakewell, 2004). The lack of research in this area led the study to explore a specific group of male consumers: adolescent male makeup users.

According to the CDMP framework, purchase desire takes place when consumers recognize a difference between their actual state and desired states (Pham, 2005). In other words, when consumers have higher desires, actual states are no longer satisfied. A wide gap between actual and desired states lead to need recognition. Marketeers continually try to create new desires in the marketplace. Need recognition is the crucial first step of the CDMP. Without going through it, marketeers will never be able to move consumers to the information search stage (Bruner, 1988). Previous research has pinpointed the difficulties in searching for and sharing information on male cosmetic products amongst adolescent males. (Poulin-Dubois, 2002; Hall, 2013). With makeup products being stereotyped as feminine in the Chinese market, need recognition of male makeup products is hard to arouse. Furthermore, even if the desire for male makeup products is aroused, it is difficult to effectively encourage prospective consumers to further explore product information. Stereotyping therefore, could significantly impact the first and second stages of the consumer decision making process.

Stereotyping is an academic tool used to analyze social psychology as well as the external influence within the context of the CDMP framework. This study will investigate whether stereotyping is an obstacle in arousing need recognition and a barrier to information search. This study will also explore the concept that the revise proportion may be true, that stereotyping is in fact a facilitator of the first two stages in the CDMP framework. This study aims to evaluate the impact of stereotyping on the purchasing behavior of male Chinese adolescents.

\section{Methodology}

Three research questions formed the basic of this study. With the aim of exploring the relationship between makeup being stereotyped as feminine and corresponding consumption behavior in the Chinese market, the following research questions were used:

RQ1: How does a conservative society maintain traditional values and form stereotypes?

RQ2: If makeup is stereotyped as being feminine, how does this affect the demand for makeup amongst male Chinese adolescents?

RQ3: If makeup is stereotyped as being feminine, how does this affect the availability of male makeup information in the Chinese market?

The phenomenological approach to analyzing qualitative data was adopted in order to gain a greater understanding of participant feelings and emotions. This approach is able to effectively reveal detailed consumer reactions (Lester, 1999; Churchill, 1985). In this study, consumer reactions to stereotyping are key to analyzing male makeup purchasing behavior in the Chinese market. In-depth interviews were conducted and prompted the researcher to explore the interviewee's inner struggles and thoughts further. Male and female stereotypes were investigated in terms of whether they were viewed as being negative or positive by 
participants and whether these views influenced the purchasing behavior or male Chinese adolescent. The phenomenological approach was of extreme benefit to not only this study but also to academics in general and markertees, as through identifying feelings and emotions, a greater understanding of the development of consumer behavior was achieved.

Discourse analysis was applied to organize interview findings (van Dijk, 1993; Jorgensen, 2002). Vocal messages and body language were analyzed to reveal male Chinese adolescent thoughts and feelings regarding stereotyping and makeup trends in the Chinese market. All findings were presented and interrupted in Microsoft Excel.

\subsection{Location and Sampling}

A total forty-seven male Chinese adolescents were interviewed: fifteen from Shanghai, sixteen from Beijing and Guangdong. Interviewees were middleclass citizens with at least two years makeup experience from tier one Chinese cities. Although sixty students applied to take part in this study, only forty-seven of them met the requirements. Interviews were conducted in English with Cantonese and Putonghua being used as supporting languages. Interview length lasted sixty to eighty minutes and interviews were conducted on using Skype or in person in Guangdong. All interviews took place from July to September 2017.

\section{Findings}

In reference to the literature review and research direction of this study, findings were divided into two parts: Stereotypes and the consumer decision making process; and stereotypes and society. The findings revealed how stereotyping aroused and influenced the first two stages of the consumer decision making process. The impact stereotyping has on Chinese society was also revealed.

\subsection{Stereotype and the Consumer Decision Making Process}

Sixty percent of participants had five to six years of experience using makeup and forty percent had two to four. Over seventy percent of them had worn makeup since secondary school. The reasons why they wore makeup were similar: they wanted to look presentable and ready for having their photo taken at any moment. One participant claimed, "It was a year long struggle to decide to wear makeup for the first. I am certain that I am not gay but boys wearing makeup used to labelled as gay, that's a big obstacle. I just simply want to look decent, why shouldn't boys be able to wear makeup?" Another participant explained, "I wanted to show off perfect skin tone and facial features which are attractive to boys, that's the reason why I started wearing makeup six years ago. I know it could be viewed as being offensive, but I think it's unhealthy to hide who we are." Another participant explained, "I began wearing makeup after my first drama performance four years ago. I looked so much better in photos where I was wearing makeup and I realized that makeup kept me looking good on camera. In our social media age where photo taking is common, I cannot stop wearing makeup. My girlfriend also supports me in wearing."

Ninety percent of participants pinpointed having a consistent appearance as being essential, so they wore makeup every day except for when they stayed at home or did sports. Moreover, 
they highlighted the need for waterproof makeup when engaging in sports to keep a perfect skin tone at all times. One participant explained, "Leaving home without wearing makeup is unacceptable to me and my male friends who also wear makeup. We think wearing makeup is a civil obligation, in the same way as we wouldn't wear pajamas on the street." Another participant pinpointed, "A lot of universities offer dress up workshops for girls where they are taught that wearing appropriate makeup is a polite gesture for different occasions. I think boys deserve professional image training too."

All participants concluded that concealer, eyebrow pencil, powder or gel were essential male makeup products. Moreover that, sixty percent wore foundation to ensure their skin was smooth and toned at all times. Others worried that foundation could be too visible resulting in a higher chance of them being labelled as homosexual. The desire for a natural look was strongly exhibited; however, their concerns regarding being labelled as homosexual constrained their purchased desires. One participant pinpointed, "I am experienced in applying natural makeup tones to my face. I would like to see the availability of more products to enhance my appearance. However, most makeup products, such as foundation, target only women, so the color options are not suitable for men." Another participant identified, "I try hard to look for proper packaging; makeup products are like clothing: they should be categorized into different groups. The functions of basic makeup products like eyebrow pencil are similar for men and women, but at least we deserve male packaging instead of dolly pink."

Findings indicated that eighty percent of participants learnt how to use makeup from online forums and friends. Sixty percent of them followed Korean makeup trends. They pinpointed that there is a lack of male Chinese makeup trends that they are proud of, which resulted in them following Korean trends. One participant pinpointed, "I learn how to apply makeup properly from my friends. It is only in the last two years, that magazines and television advertisements have begun to concentrate more on male makeup products. In addition, we do not have much choice when it comes to makeup products, so product application demos for men are rare." Another participant explained, "The Korean makeup trends have definitely affected me, Korea music and drama have influenced what I consider to be attractive. Also, their makeup skills are extremely detailed." One more participant mentioned, "I like both Korean and American male makeup styles; however, I think an individual's makeup style is personal to them, I just want to present myself in a decent way and I guess the tendency to look natural by using makeup is more popular in China."

\subsection{Stereotype and the Society}

Findings indicated that ninety percent of participants experienced negativity after wearing makeup. The belief that wearing makeup is feminine is strong in Mainland China. The emergence of a male makeup trend has led to confusion not only amongst participants but also within Chinese society. However, the difficulties faced by participants did not prevent them using male makeup. In striking contrast, it encouraged them to consider the impact on others rather than just focusing on themselves. One participant identified, "I can ignore all the questions except those from my mother. She feels ashamed when relatives ask if I am gay. At 
the same time, although she is against the idea of me wearing makeup, she loves me and is therefore prepared to defend my sexuality to others; it's a painful process. It took me two years to explain to my mother clearly that wearing makeup is a lifestyle choice, like choices made by working women in presenting a professional image." Another participant explained, "Wearing makeup has made me a better person, more mature and considerate of others. Although I am brave enough to wear makeup in Mainland China, I still have to be aware and sensitive to the fact that I may have offended my parents or teachers. I felt good when I was in Korea. Wearing male makeup is the norm there. People did not stare at me and no one questioned why I was wearing makeup or my sexuality like they would do in Mainland China."

The findings suggested that male Chinese adolescents are not ready to share their makeup opinions and skills on social media. Surprisingly, they were concerned not only about social norms but also their ability in applying makeup. Even though they have certain concern regarding the attitude of others regarding makeup products being labelled as feminine. They pinpointed that social media is a platform to express personal feelings and the healthy development of male make up trends. One participant explained, "My social media photos are acceptable in Chinese society: tidy and decent with natural makeup. Unlike male makeup advertisements that use homosexual looking artists to present their products. This is why people in our society link male makeup use with homosexuality." Another participant stated, "If my makeup skills get to a certain level, I would love to become a male makeup blogger. I think social media could be a good platform from which to spread the trend and increase acceptance levels." Another participant highlighted, "Well, it's not easy for me to initiate discussions on this topic. I do not want the attention, especially on social media. Also, I do not think that society is ready to accept the whole concept of male makeup, but I guess if other males begin the discussion, I will join in."

\section{Discussions}

This study pinpointed stereotype revealed the desire of male makeup consumption in Mainland China. Findings revealed that even though wearing makeup was stereotyped as being feminine in Mainland China, it did not weaken the desire of male Chinese adolescents for such products. Interestingly, these negative challenges could in fact be seen as encouraging a new male makeup trend. In addition, the strong desire of male Chinese adolescents for makeup products is causing male and female stereotype to evolve in the Chinese market, which resulting in a significant area of potential study for marketeers.

\subsection{The Aroused of New Desires in Conservative Market as a Result of Stereotyping}

Findings identifies that the deep rooted traditions had a considerable impact on male Chinese adolescents. The stereotyping of makeup as being feminine leads society to label those males who use makeup as being non-conforming. The anxiety this causes male Chinese adolescent makeup users affects not only the users themselves but also their family and trends. Even though male Chinese adolescents who wear makeup have adopted male makeup as a lifestyle, they still wish to diminish the negative influence this has on others around them. They acknowledged that there was a contradiction between their appearance after using makeup 
and the traditional images of males available in Mainland China. At the same time, they are trying to push the boundaries between what is acceptable in their society and what is not, in terms of male makeup use in order to satisfy their desires. Their desire to wear makeup was not aroused by promotional materials. Surprisingly, findings indicated that women were stereotyped as needing to be presentable at all times. Participants opined that this stereotype should not just be limited by gender. They pinpointed the female desire for perfect skin tones and ordinary appearance as even being a requirement in presenting a professional image. In addition, universities and marketeers offered free training and demos for females wishing to understand more about how to use makeup. Moreover, participants questioned the belief that male makeup use indicated sexual orientation. With this in mind, the stereotype of makeup being feminine was found to be being challenged by non-conforming members: male Chinese adolescents. These members are instrumented in evolving the stereotype. At the same time, their deep rooted Chinese culture has led them to balance desire with tradition in their society.

\subsection{Lack of Available Information in Conservative Market Due to Stereotype}

Findings pinpointed that the lack of product choice and related information in society has strengthened the belief that male makeup usage is abnormal and users are part of a non-conforming group. The stereotype of makeup being feminine in society challenged the participant's desire for such products due to lack of available information on the market. They desire proper male packaging for makeup products and product enquiry counters. Their strong desire to become recognized as a segment in the makeup market has been strongly exhibited. Participants concluded that makeup usage is a part of their daily life. A lack of information on male makeup products strengthened the belief amongst participants that they were not properly recognized either by their society or by the makeup industry as a whole. Findings concluded that current socio-cultural influences and stereotypes have confused male Chinese adolescent makeup consumption: the confusion between actual state and desired state; and confusion caused as a result of not knowing there place in society.

The lack of male makeup information and deep rooted Chinese traditions were found to be a challenge for participants. However, such challenges were found to be positive rather than negative. Participants displayed a strong desire to be satisfied, seeking male makeup information from both Korea and America. By mimicking Korean makeup styles, male Chinese adolescents are able to explore unique makeup styles for themselves. Also, they desire to breach the stereotype that makeup use is only for females and male makeup users must be homosexual. They admired and respected their own deep rooted culture just like others citizens of the world. The contradiction between their strong desires to use makeup and the image of the traditional male in their society did not lead them to blindly follow either a Korean or an American trend. Instead, they are nurturing their own unique makeup trends in Mainland China.

\subsection{Implications for Academics and Marketeers}

Previous research has pinpointed non-conforming members as encountering anxiety due to strong stereotyping in their respective societies. It has also identified reactions to stereotyping as indicating how consumers respond in their societies. Not only was it concluded that 
stereotyping was a significant indicator of their reactions to society, but the previous research also identified stereotyping as having a negative socio-cultural impact. Conversely, in this study, stereotyping was identified as having a positive impact on male Chinese adolescent makeup consumption. Even though male and female stereotyping caused confusion amongst male makeup users during the consumption process, it singled them out as non-conforming members. This motivated them to speak out and challenge the traditional male and female stereotypes in the Chinese market.

In conclusion and in line with previous studies, this study put forward the need for communication amongst male Chinese adolescents, makeup product providers and their respective societies. In reference to the consumer decision making process, findings showed that the need recognition and information search stages were weakened by male and female stereotyping in the Chinese market. In addition, it showed two significant points for academics and marketeers to note: male Chinese adolescents are not only a rising market segment but they are not a clearly defined group yet and their needs are not satisfied. Proper ways of communicating with them through promotional wordings and packaging are crucial to encourage purchase. Therefore, academics need to carry out more research on such segments to explore their characteristics as well as deepen and widen understanding on the behavior and development of such consumers. Marketeers should firstly try to understand the current needs of such potentially highly lucrative market segment. After that, they should try to develop make up trends together with male Chinese adolescents. By responding to their needs and wants also, they need to be aware of and incorporate typical traditional conservative values associated with the Chinese market when communicating with male Chinese adolescents.

\section{Limitations and Future Research Directions}

This research explored the impact of stereotyping on purchasing. There are two major limitations of this study, both regarding external validity: samples did not represent the entire Mainland Chinese population, there is a lack of research on male makeup consumption. This study did not come about as a result of previous studies, instead, it explored male makeup as a new component of the male purchase decision framework. These limitations open the door for future research and suggest male makeup usage as a potential new study area.

Firstly, previous research has identified the existence of negative impact of stereotyping male cosmetic products. Socio-cultural and personal factors were a key focus of previous research regarding the relationship between stereotyping and the purchase decision. In this study, relevant socio-cultural factors that influenced stereotyping were selected and analyzed in terms of how the purchase decision was affected. In addition, this study was further narrowed down by three criteria, depending on which first-tier cities interviewees were selected from: Beijing, Shanghai or Guangzhou. While first-tier citizenship was the first requirement of this study, the selection criteria also included at least two years makeup experience and being from a middleclass family. The sample could not represent the entire Mainland Chinese market, as it was only representative of male Chinese adolescents in first-tier cities. Also, their families economic status and makeup experience were similar, from which generalize 


\section{Macrothink}

inferences could be made. This study identified stereotyping as brining certain positive outcomes from respondents despite operating within a traditionally negative stereotypical environment. The anxiety and confusion they encountered during the purchase decision can be further explored within the context of other variables: ethnicity, sexuality and self-image.

Secondly, this research focused on a new area of study: male makeup purchasing behavior. Another limitation regarding external validity was the lack of research on male makeup consumption. The previous research in male cosmetic consumption was too broad to be used as a reference for this study. Makeup was seen as being just another cosmetic item and in some instances not even included in previous research. As male consumption is an emerging consumer segment, especially in cosmetics, male makeup as a specific area is a worthwhile field of study. Without strong reference from previous research, this study laid the foundation for further research on male makeup and its constraints in a conservative market.

In conclusion, the limitations of this study open the door for a multitude of potential research in the future. Not only were the two limitations worthy of deeper exploration, the conclusion of this study pinpointed the need to allow for traditional values and the emergence of conflicting desires in a conservative market. Exploring and stimulating new desires is the key objective of marketeers; however, allowing for traditional values could minimize the confusion of consumers when experiencing new desires for new products. The study of this relationship is worthwhile from a point of view of both market development and the understanding of corresponding consumer behavior.

Set of questions used to determine participants

1. Which province did you grow up in?

2. How long have you been wearing makeup?

3. How would you describe your family's economic status: very rich, rich, average, poor, very poor?

Interview questions

Part 1

1. When did you start wearing makeup? Why?

2. How often do you wear makeup?

3. What kind of makeup do you wear? Why?

4. How did you learn how to use makeup?

5. Do you like Korean and or American makeup trends? If yes, have they influenced what makeup you use and your individual style? 
Part 2

1. Have you ever been discouraged from using or questioned about wearing makeup?

2. Will you share makeup ideas and or product information with your friends on social media? Why?

3. Is it difficult to find makeup product information for males in Mainland China?

4. Do you think society has labelled makeup as being feminine? If yes, will that influence your decision to wear makeup?

5. Do you think society accepts men wearing makeup?

\section{References}

Bakewell, C. M.-W. (2004). Male Consumer Decision-Making Styles. The International Review of Retail, Distribution and Consumer Research, 223-240. https://doi.org/10.1080/0959396042000178205

Banse, R. G. (2010). The development of spontaneous gender stereotyping in childhood: Relations to stereotype knowledge and stereotype flexibility. Developmental Science, 298-306. https://doi.org/10.1111/j.1467-7687.2009.00880.x

Bruner, G. C. (1988). Problem Recognition: the Crucial First Stage of the Consumer Decision Process. Journal of Services Marketing, 43-53. https://doi.org/10.1108/eb024733

Carter, J. D. (2006). Individual differences in the acceptance of stereotyping. Journal of Research in Personality, 1103-1118. https://doi.org/10.1016/j.jrp.2005.11.005

Churchill, S. D. (1985). An Introduction to Phenomenological Psychological for Consumer Research: Historical, Conceptual, and Methodological Foundations. Advances in Consumer Research, 550-555.

Clow, K. E. (2005). CONSUMER DECISION-MAKING PROCESS. Concise Encyclopedia of Advertising, 44-45.

Cohen, G. L. (2005). "I Am Us": Negative Stereotypes as Collective Threats. Journal of Personality and Social Psychology, 566-582. https://doi.org/10.1037/0022-3514.89.4.566

Consumer Behavior: Buying, Having, and Being (3rd ed.). (1998). Psychology \& Marketing.

Cuddy, A. J. (2015). Men as cultural ideals: Cultural values moderate gender stereotype content. Journal of Personality and Social Psychology, 622. https://doi.org/10.1037/pspi0000027

Dahl, D. (2014). Social influence and consumer behavior. Journal of Consumer Research, S1-S2.

de Vries, D. A. (2014). The Effect of Social Network Site Use on Appearance Investment and Desire for Cosmetic Surgery Among Adolescent Boys and Girls. Sex Roles, 283-295. https://doi.org/10.1007/s11199-014-0412-6 
Fiske, S. T. (1989). Examining the role of intent: Toward understanding its role in stereotyping and prejudice. In Unintended thought. (pp. 253-283).

(1989). Examining the role of intent: Toward understanding its role in stereotyping and prejudice. In S. T. Fiske, Unintended thought. (pp. 253-283).

(1998). Stereotyping, prejudice, and discrimination. In S. T. Fiske, Handbook of social psychology (pp. 357-411).

Hall, M. G.-S. (2013). Stake Management in Men's Online Cosmetics Testimonials. Psychology and Marketing, 227-235. https://doi.org/10.1002/mar.20600

Hopkins, B. E. (2007). Western cosmetics in the gendered development of consumer culture in China. Feminist Economics, 287-306. https://doi.org/10.1080/13545700701439416

Jeddi, S. (2013). Consumer behavior and Consumer buying decision process. International Jornal of Business and Behavioral Sciences, 20-23.

Jorgensen, M. P. (2002). Discourse Analysis as Theory and Method. Theory, 229. https://doi.org/10.4135/9781849208871

Khan, I. D. (2017). Men's attitude and motivation toward consumption of grooming products: A comparison of Chinese and Pakistani male consumers. Cogent Business \& Management, $1-14$.

Kumar, H. H. (2014). A Study on factors influencing consumer buying behavior in cosmetic Products. International Journal of Scientific and Research Publications, 2250-3153.

Lee, K. K. (2011). Stereotype Threat in the Marketplace: Consumer Anxiety and Purchase Intentions. Journal of Consumer Research, 343-357. https://doi.org/10.1086/659315

Lester, S. (1999). An introduction to phenomenological research. Retrieved February, 1-4.

Leung, K. M. (2015). Values, schemas, and norms in the culture-behavior nexus: A situated dynamics framework. Journal of International Business Studies, 1028-1050. https://doi.org/10.1057/jibs.2014.66

Li, L. K. (2009). Sexuality Education in China: The conflict between Reality and Ideology. Asia Pacific Journal of Education, 469-480. https://doi.org/10.1080/02188790903309066

Luo, Y. (2009). Analysis of Culture and Buyer Behavior in Chinese Market. Asian Culture and History, 25-31. https://doi.org/10.5539/ach.v1n1p25

Luoh, H.-F. T.-H. (2009). Physical attractiveness stereotypes and service quality in customer-server encounters. The Service Industries Journal, 1093-1104. https://doi.org/10.1080/02642060902764517

Morphitou, R. T. (2011). A Consumer Behavior Analysis of the Cosmetic Industry in Cyprus: the Case of the Seventeen Brand. International Journal of Management Cases, 200-202. https://doi.org/10.5848/APBJ.2011.00053 
Oakes, P. J. (1994). Stereotyping and social reality. Stereotyping and social reality.

Parekh, H. K. (1994). The physical attractiveness stereotype in a consumer-related situation. The Journal of Social Psychology, 297-300. https://doi.org/10.1080/00224545.1994.9711733

Pellémans, P. A. (1971). The consumer decision - making process. European Journal of Marketing, 8-21. https://doi.org/10.1108/EUM0000000005155

Pham, M. T. (2005). Promotion and prevention in consumer decision-making: State of the art and theoretical propositions.

Poulin-Dubois, D. S. (2002). Men don't put on make-up: Toddlers' knowledge of the gender stereotyping of household activities. Social Development, 166-181. https://doi.org/10.1111/1467-9507.00193

Ricciardelli, R. C. (2009). Men, appearance, and cosmetic surgery: The role of self-esteem and comfort with the body. Canadian Journal of Sociology, 105-134.

Rieder, E. A. (2015). Men and Cosmetics: Social and Psychological Trends of an Emerging Demographic. Journal of drugs in dermatology : JDD, 1023-1026.

Rocha, M. A. (2005). Age, gender and national factors in fashion consumption. Journal of Fashion Marketing and Management: An International Journal, 380-390. https://doi.org/10.1108/13612020510620768

Roest, A. M. (2009). Value similarities among fathers, mothers, and adolescents and the role of a cultural stereotype: Different measurement strategies reconsidered. Journal of Research on Adolescence, 812-833. https://doi.org/10.1111/j.1532-7795.2009.00621.x

Siyanova-Chanturia, A. W. (2015). Gender stereotypes across the ages: On-line processing in school-age children, young and older adults. Frontiers in Psychology. https://doi.org/10.3389/fpsyg.2015.01388

Souiden, N. D. (2009). Canadian and French men's consumption of cosmetics: a comparison of their attitudes and motivations. Journal of Consumer Marketing, 97-109. https://doi.org/10.1108/07363760910940465

Spencer, S. J. (2016). Stereotype Threat. Annual Review of Psychology, 415-437. https://doi.org/10.1146/annurev-psych-073115-103235

van Dijk, T. A. (1993). Principles of Critical Discourse Analysis. Discourse \& Society, 249-283. https://doi.org/10.1177/0957926593004002006

Weber, J. M. (2002). Differences in purchase behavior between France and the USA: the cosmetic industry. Journal of Fashion Marketing and Management, 396-407. https://doi.org/10.1108/13612020210448673

Zhou, L. W. (2014). Social media: A new vehicle for city marketing in china. Cities, 27-32. https://doi.org/10.1016/j.cities.2013.11.006 


\section{Macrothink}

Journal of Social Science Studies

ISSN 2329-9150

\section{Copyright Disclaimer}

Copyright for this article is retained by the author(s), with first publication rights granted to the journal.

This is an open-access article distributed under the terms and conditions of the Creative Commons Attribution license (http://creativecommons.org/licenses/by/3.0/). 